\title{
THE SECOND COEFFICIENT OF A FUNCTION WITH ALL DERIVATIVES UNIVALENT
}

\author{
A. SATHAYE and S.M. SHAH \\ Department of Mathematics \\ University of Kentucky \\ Lexington, Kentucky 40506 U.S.A. \\ (Received July 7, 1981 and in revised form December 9, 1981)
}

ABSTRACT. We consider the second coefficient of a class of functions, univalent and normalized, and with all derivatives univalent in the unit disk $D$, and improve on a known result. It is also shown that this bound is in a sense best possible. KEY WORDS AND PHRASES. Univalent functions, Typically real functions, Entire functions of exponential type.

1980 MATHEMATICS SUBJECT CLASSIFICATION CODES. Primary 30C50, Secondary 30015.

1. INTRODUCTION.

Let $S$ be the family of functions of the form

$$
f(z)=z+\sum_{k=2}^{\infty} a_{k} z^{k}
$$

which are analytic and univalent in the unit disk D: $|z|<1$. Let E be the subfamily of $S$ such that if $f \in E$, then each $f^{(n)}$ is univalent in $D, n=0,1,2, \ldots$. Set

$$
\alpha=\sup \left\{\left|\mathrm{a}_{2}\right|: \mathbf{f} \in \mathrm{E}\right\} \text {. }
$$

Then it is known that $f$ must be an entire function of exponential type not exceeding $2 \alpha[2,3]$ and $[1,2]$

$$
1.5910 \leq \alpha<1.7208 \text {. }
$$

We construct, in Section 2, a function $f$ belonging to $E$ for which

$$
a_{2}=\frac{f^{\prime \prime}(0)}{2}>\frac{3.18781}{2}=1.593905 \text {. }
$$


Consider now a subfamily of $\mathrm{E}, \mathrm{E}_{+}$defined as follows:

$$
E_{+}=\left\{f \mid f \in E, f^{(k)}(0)>0, k=1,2, \ldots\right\} \text {. }
$$

Set

$$
\alpha_{+}=\sup \left\{a_{2}, f \in E_{+}\right\}
$$

It follows from an unpublished result of Ted Suffridge that

$$
\alpha_{+}<1.62031 \text {. }
$$

Note that the function $f$ defined below by (2.2) belongs to $E_{f}$, and so a lower bound for $\alpha_{+}$is given by $(1.4)$.

2. MAIN RESULT.

In the following we shall write

$$
\zeta=\left\{(T+\pi) e^{-\pi}+(T-\pi) e^{\pi}\right\} / 2
$$

where $T$ is a positive constant.

THEOREM. Let

$$
\begin{aligned}
\phi(z)= & \{\exp (\pi(1+z))-\exp (-\pi(1+z))\} / 2 \pi+A z \\
& +(A T+\zeta) \frac{z^{2}}{2}+B z^{3}
\end{aligned}
$$

where $\mathrm{A}, \mathrm{B}, \mathrm{T}$ are nonnegative constants, and let

$$
f(z ; A, B, T) \equiv f(z)=\{\phi(z)-\phi(0)\} / \phi^{\prime}(0) .
$$

(i) If $\mathrm{T}=3.18781018, \mathrm{~A}=0.03666, \mathrm{~B}=0$ then $\mathrm{f}$ defined by $(2.2)$ belongs to E and

$$
2 \mathrm{a}_{2}=\mathrm{f}^{\prime \prime}(0)=\mathrm{T}>3.18781 \text {. }
$$

(ii) If $\mathrm{T}=3.18782$ then $\mathrm{f}$ defined by (2.2) does not belong to $\mathrm{E}$ for any choice of nonnegative constants A, B.

PROOF. We require the following:

DEFINITION. Let $f(z)$ be regular in D. Then $F(z)$ is called typically-real if and only if

$$
\operatorname{Im} f(z)=0 \Leftrightarrow \operatorname{Im} z=0
$$

The following theorem is due to S.Y. Trimble.

THEOREM A [4]. Let $f(z)=z+\sum_{n=2}^{\infty} a_{n} z^{n}$ be regular in $D$ and suppose that all $a_{n}$ are real. Then a necessary and sufficient condition that $f \in E$ is that each 
$\mathrm{f}^{(\mathrm{k})}(\mathrm{k}=0,1,2, \ldots)$ is typically real in $\mathrm{D}$.

PROOF OF (i). It is easily seen that $\phi(0)=\frac{\sinh \pi}{\pi}>0, \phi^{\prime}(0)=\cosh \pi+A>0$; and

$$
f(z)=z+\frac{T}{2} z^{2}+\left\{\left(\left(e^{\pi}+e^{-\pi}\right) \frac{\pi^{2}}{12}+B\right) /\left(\frac{e^{\pi}+e^{-\pi}}{2}+A\right)\right\} z^{3}+\ldots
$$

is entire and of exponential type $\pi$ and has all positive coefficients. Further, writing $z=x+i y$, we have

$$
\left\{\frac{\operatorname{Im} \phi^{\prime}(z)}{y}\right\}=\frac{\sin \pi y \sinh \pi(1+x)}{y}+(A T+\zeta)+6 B x .
$$

Write

$$
\psi_{2}(x)=\left(\frac{\operatorname{Im} \phi^{\prime}(z)}{\operatorname{Im} z}\right)|z|=1 .
$$

Then

$$
\psi_{2}(x)=\frac{\sin \pi \sqrt{1-x^{2}}}{\pi \sqrt{1-x^{2}}} \pi \sinh \pi(1+x)+(A T+\zeta)+6 B x .
$$

This function $\psi_{2}$ is positive for $-1 \leq x \leq 1$, since $0=6 \mathrm{~B}<\mathrm{AT}+\zeta$ by our choice of T, $A$ and B. Hence $\phi^{\prime}(z)$ is typically real. Further,

$$
\phi^{\prime \prime}(z)=\pi \sinh \pi(1+z)+A T+\zeta+6 B z,
$$

and since $B=0$

$$
\text { Im } \phi^{\prime \prime}(z)=\pi \sinh \pi y \cosh \pi(1+x) .
$$

This shows that $\phi^{\prime \prime}(z)$ is typically real. Similarly each $\phi^{(k)}(z), k=3,4, \ldots$ is typically real.

Consider now

$$
\begin{aligned}
\psi_{1}(x) & =\left(\frac{\operatorname{Im} \phi}{\operatorname{Im} z}\right)|z|=1 \\
& =\{\cosh \pi(1+x)\} \frac{\sin \pi \sqrt{1-x^{2}}}{\pi \sqrt{1-x^{2}}}+A+(A T+\zeta) x+B\left(4 x^{2}-1\right) .
\end{aligned}
$$

Note that $\psi_{1}(x)>0$ for $0 \leq x \leq 1$. Write now $F(x)=\psi_{1}(-x)$. Then

$$
F(x)=\{\cosh \pi(1-x)\} \frac{\sinh \pi \sqrt{1-x^{2}}}{\pi \sqrt{1-x^{2}}}+A-(A T+\zeta) x+B\left(4 x^{2}-1\right) .
$$

Write

$$
\begin{array}{ll}
C(x)=\cosh \pi(1-x), & \left.H(x)=\left(\sinh \pi \sqrt{1-x^{2}}\right) / \pi \sqrt{1-x^{2}}\right) \\
G(x)=A-(A T+\zeta) x, & S(x)=\sinh \pi(1-x) .
\end{array}
$$


Then since $B=0$,

$$
\begin{aligned}
F(x) & =C(x) H(x)+G(x), \\
F^{\prime}(x) & =\{-\pi \sinh \pi(1-x)\} H(x)+C(x) H^{\prime}(x)+G^{\prime}(x),
\end{aligned}
$$

and

$$
H(x)=x^{2} \prod_{n=2}^{\infty}\left(\frac{n^{2}-1+x^{2}}{n^{2}}\right) .
$$

Now $B=0$ and for $0 \leq x \leq 0.046<\frac{A}{(A T+\zeta)}, G(x)=A-(A T+\zeta) x>0$.

Further for $x \in[0.92,1]$ we have

and

$$
\begin{aligned}
& \min C(x)=C(1)=1, \\
& \min H(x)=H(0.92)>0.765 \\
& \min G(x)=G(1)>-0.752 .
\end{aligned}
$$

Thus $F(x)>0$ for $x \in[0.92,1]$ and also for $x \in[0,0.04]$.

Differentiating $\log \mathrm{H}(\mathrm{x})$, we have

$$
\frac{H^{\prime}(x)}{H(x)}=\frac{2}{x}+2 x \sum_{n=2}^{\infty} \frac{1}{n^{2}-1}-\varepsilon(x)
$$

where

$$
\varepsilon(x)=\sum_{n=2}^{\infty}\left(2 x^{3}\right) /\left\{\left(n^{2}-1\right)\left(n^{2}-1+x^{2}\right)\right\} .
$$

Write $u(x)=(2 / x)+(3 x / 2)$. Then

$$
F^{\prime}(x)=-\pi S(x) H(x)+C(x) u(x) H(x)-\varepsilon(x) C(x) H(x)-(A T+\zeta) .
$$

Now $0 \leq \varepsilon(x)<(0.27) x^{3}$ in the interval $(0.040,1)$. Further in this interval, $\mathrm{u} \downarrow, \mathrm{C} \downarrow, \mathrm{S} \downarrow, \mathrm{H}^{\prime}>0$ and so $\mathrm{H} \uparrow$. Now $\mathrm{u}(1)>0$ and so $\mathrm{u}(\mathrm{x})>0, \mathrm{C}(1)=1$ and so $C(x)>0, S(1)=0$ and so $S(x)>0, H(x) \rightarrow 1$ as $x \rightarrow 1-0, H(0)=0, H(x)>0$ and $\varepsilon(x)<0.27 x^{3}$ and $x^{3} \uparrow$ and positive.

Let $x_{1} \leq x \leq x_{2}$ where $x_{1}, x_{2}$ belong to this interval. Denote by subscript 2 the value of these functions at $x_{2}$ and by subscript 1 at $x_{1}$. Let

$$
\begin{aligned}
& \mathrm{T}_{1}\left(\mathrm{x}_{1}, \mathrm{x}_{2}\right) \equiv \mathrm{T}_{1}=\mathrm{u}_{2} \mathrm{C}_{2} \mathrm{H}_{1}-\mathrm{H}_{2}\left(\pi \mathrm{S}_{1}+\mathrm{C}_{1}\left(0.27 \mathrm{x}_{2}^{3}\right)\right)-(\mathrm{AT}+\zeta), \\
& \mathrm{T}_{2}\left(\mathrm{x}_{1}, \mathrm{x}_{2}\right) \equiv \mathrm{T}_{2}=\mathrm{u}_{1} \mathrm{C}_{1} \mathrm{H}_{2}-\mathrm{H}_{1}\left(\pi \mathrm{S}_{2}\right)-(\mathrm{AT}+\zeta) .
\end{aligned}
$$

Then for $\mathrm{x}_{1} \leq \mathrm{x} \leq \mathrm{x}_{2}, \mathrm{~T}_{1}<\mathrm{F}^{\prime}(\mathrm{x})<\mathrm{T}_{2}$. Hence we have

PROPOSITION 2. If $\mathrm{T}_{1}>0$ or $\mathrm{T}_{2}<0$ then $\mathrm{F}(\mathrm{x})$ is monotone in this interval and so if $F\left(x_{1}\right)>0, F\left(x_{2}\right)>0$ then $F(x)$ is positive. 
COROLLARY 3. Suppose that the quantities $\left\{\mathrm{T}_{i}\right\}$ and $\left\{F\left(\mathrm{x}_{i}\right)\right\}$ are machine calculated with at least 6-digit accuracy and we have:

(i) $\mathrm{T}_{1} \geq 10^{-6}$ or $\mathrm{T}_{2} \leq-10^{-6}$,

(ii) $\quad F\left(x_{1}\right)>10^{-6}, F\left(x_{2}\right)>10^{-6}$

then $F(x)$ is positive in $\left[x_{1}, x_{2}\right]$.

COROLLARY 4. By machine calculations we have:

(i) For

$$
\begin{aligned}
& {\left[\mathrm{x}_{1}, \mathrm{x}_{2}\right]=[0.040000,0.054844]} \\
& \mathrm{T}_{2}=-0.0410121, \quad \mathrm{~F}\left(\mathrm{x}_{1}\right)=0.0133178 \\
& \mathrm{~F}\left(\mathrm{x}_{2}\right)=0.0081411
\end{aligned}
$$

(ii) For

$$
\begin{aligned}
& {\left[x_{1}, x_{2}\right]=[0.847885,0.918943]} \\
& T_{1}=0.0373463, \quad F\left(x_{1}\right)=0.0354489 \\
& F\left(x_{2}\right)=0.1001402
\end{aligned}
$$

(iii) For

$$
\begin{aligned}
& {\left[\mathrm{x}_{1}, \mathrm{x}_{2}\right]=[0.918943,0.990000]} \\
& \mathrm{T}_{1}=0.8434650, \quad \mathrm{~F}\left(\mathrm{x}_{1}\right)=0.1001402 \\
& F\left(\mathrm{x}_{2}\right)=0.2242320 .
\end{aligned}
$$

Hence $F(x)$ is positive in the intervals $(2.6),(2.7)$ and (2.8). Thus it is now enough to show that $F(x)>0$ in $[0.054844,0.847885]=\left[X_{1}, X_{2}\right]$ say. We now consider when $\mathrm{T}_{1}<0, \mathrm{~T}_{2}>0$. Write $\mathrm{M}\left(\mathrm{x}_{1}, \mathrm{x}_{2}\right) \equiv \mathrm{M}=-\mathrm{T}_{1} \cdot\left(\mathrm{x}_{2}-\mathrm{x}_{1}\right)$. By the Mean-Value Theorem, $F\left(x_{0}\right)=F\left(x_{1}\right)+F^{\prime}(\xi)\left(x_{0}-x_{1}\right)$, where $x_{1} \leq x_{0} \leq x_{2}$ and $\xi \epsilon\left(x_{1}, x_{0}\right)$. Hence

$$
\begin{aligned}
F\left(x_{0}\right) & \geq F\left(x_{1}\right)+T_{1} \cdot\left(x_{0}-x_{1}\right) \\
& >F\left(x_{1}\right)+T_{1} \cdot\left(x_{2}-x_{1}\right)=F\left(x_{1}\right)-M
\end{aligned}
$$

for every $x_{0}$ in $\left[x_{1}, x_{2}\right]$. Hence we have

PROPOSITION 5. Let $\mathrm{T}_{1}<0$ and $\mathrm{F}\left(\mathrm{x}_{1}\right)>0, \mathrm{~F}\left(\mathrm{x}_{2}\right)>0$. If $\mathrm{F}\left(\mathrm{x}_{1}\right)>\mathrm{M}\left(\mathrm{x}_{1}, \mathrm{x}_{2}\right)$ then $F(x)>0$ in $\left[x_{1}, x_{2}\right]$.

COROLLARY 6. Suppose that $\left\{\mathrm{F}\left(\mathrm{x}_{1}\right)\right\}$ and $\mathrm{M}\left(\mathrm{x}_{1}, \mathrm{x}_{2}\right)$ are machine calculated with at leat 6-digit accuracy and we have 
(i) $\quad F\left(x_{1}\right)>10^{-6}, \quad F\left(x_{2}\right)>10^{-6}$

(ii) $\quad F\left(x_{1}\right)>M\left(x_{1}, x_{2}\right)+10^{-6}$

then $F(x)$ is positive in $\left[x_{1}, x_{2}\right]$.

PROOF THAT $F(x)>0$ IN $\left[x_{1}, x_{2}\right]$. This was carried out by running the following simple program and repeatedly applying Corollary 6, as indicated.

(1) Set $x_{1}=x_{1}, x_{2}=x_{2}$.

(2) Apply test of Corollary 6. Is the hypothesis satisfied? If not, then go to (5).

(3) $\quad F(x)$ is now positive in $\left[x_{1}, x_{2}\right]$. Is $x_{2}-x_{2}<10^{-6}$ ? If it is, then stop. We have proved $F(x)>0$ in $\left[x_{1}, x_{2}\right]$ and $x_{2}$ is as close to $x_{2}$ as is reasonable. If $x_{2}-x_{2} \geq 10^{-6}$, then continue.

(4) Start with the next interval by replacing $x_{1}$ by $x_{2}$ and $x_{2}$ by $x_{2}$. Go to (2).

(5) $\left[\mathrm{x}_{1}, \mathrm{x}_{2}\right]$ is too large. Replace $\mathrm{x}_{2}$ by $\left(\mathrm{x}_{1}+\mathrm{x}_{2}\right) / 2$. Is $\left(\mathrm{x}_{2}-\mathrm{x}_{1}\right)<10^{-6}$ ? If it is, then stop. This method cannot be reliably continued beyond $x_{1}$. If $x_{2}-x_{1} \geq 10^{-6}$, then go to (2).

This program terminated by $x_{2}$ reaching $x_{2}$. In fact the program was run from 0.04 to 0.99 by incorporating the test of Corollary 4 , as well as another mean value test comparing $F\left(x_{2}\right)$ and $T_{2} \cdot\left(x_{2}-x_{1}\right)$ when $T_{2}>0$. The numbers presented here were generated by the program and are not intrinsically significant. The table for the relevant calculations is available upon request.

This completes the proof of the statement that $F(x)>0$ in $-1 \leq x \leq 1$ and hence that of (i).

(ii) We require the following:

LERMA. Consider a family of functions of two parameters $(a, b)$ defined by

$$
\phi(x, a, b)=a f(x)-b g(x)+h(x) .
$$

Suppose that there exist $a_{0}, x_{1}, x_{2}$ satisfying

$$
\begin{gathered}
\phi\left(x_{i}, a_{0}, 0\right)<0, \quad i=1,2, \\
f\left(x_{1}\right), g\left(x_{1}\right)>0 ; f\left(x_{2}\right), g\left(x_{2}\right)<0,
\end{gathered}
$$


and

$$
f\left(x_{1}\right) / g\left(x_{1}\right) \leq f\left(x_{2}\right) / g\left(x_{2}\right) .
$$

Then for $a l l a, b \geq 0$ we must have either

$$
\left(\mathrm{x}_{1}, \mathrm{a}, \mathrm{b}\right)<0 \text { or }\left(\mathrm{x}_{2}, \mathrm{a}, \mathrm{b}\right)<0 .
$$

REMARK. In this lemma and proof of (ii), $f$ is different from $f$ in (2.2).

PROOF. Assume the contrary. We will show that the resulting system of inequalities has no solution. Set $\alpha=a-a_{0}, \beta=b$. Then we get from (2.10) and our assumption,

$$
0<\phi\left(x_{i}, a, b\right)-\phi\left(x_{i}, a_{0}, 0\right)=\alpha f\left(x_{i}\right)-\beta g\left(x_{i}\right), \quad i=1,2 .
$$

Also by our assumption

$$
\beta=b \geq 0 \text {. }
$$

From (2.15), (2.14) and (2.11) we get $\alpha \geq 0$. Now we claim that $\beta \neq 0$; for otherwise (2.14) (with $i=2$ ) and (2.11) give $\alpha f\left(x_{2}\right)>0$, that is $\alpha<0$ leading to a contradiction.

Thus $\beta>0$ and from (2.14) (with $i=1$ ) and (2.11) we get $\alpha>0$. Now (2.14) (with $i=1,2$ ) and $(2.11$ ) yield

$$
\frac{f\left(x_{1}\right)}{g\left(x_{1}\right)}>\frac{\beta}{\alpha}>\frac{f\left(x_{2}\right)}{g\left(x_{2}\right)}
$$

in contradiction to (2.12). The lemma is proved.

PROOF OF (ii). Let, in the lemma,

$$
\begin{aligned}
& \phi(x, a, b)=C(x) H(x)-\zeta x+a(1-T x)-b\left(1-4 x^{2}\right), \\
& a=A, \quad b=B, \quad f(x)=1-T x, \quad g(x)=1-4 x^{2}, T=3.18782, \\
& h(x)=C(x) H(x)-\zeta x .
\end{aligned}
$$

Then from (2.5) and (2.9) we see that

$$
\phi(\mathrm{x}) \equiv \phi(\mathrm{x}, \mathrm{a}, \mathrm{b})=\phi(\mathrm{x}, \mathrm{A}, \mathrm{B})=\mathrm{F}(\mathrm{x}) .
$$

Let $x_{1}=0.1160000, x_{2}=0.7033334, a_{0}=0.0366100$. Then the following are correct up to 6 decimals at least.

$$
\begin{aligned}
& \phi\left(\mathrm{x}_{1}\right) \stackrel{2}{=}-0.0000062, \phi\left(\mathrm{x}_{2}\right)=-0.0000037, \\
& \mathrm{f}\left(\mathrm{x}_{1}\right)=1-\mathrm{Tx}_{1}=0.6302128, \mathrm{f}\left(\mathrm{x}_{2}\right)=-1.2421002,
\end{aligned}
$$




$$
\begin{aligned}
& g\left(x_{1}\right)=1-4 x_{1}^{2}=0.9461760, g\left(x_{2}\right)=-0.9787114, \\
& \frac{f\left(x_{1}\right)}{g\left(x_{1}\right)}=0.6660630, \frac{f\left(x_{2}\right)}{g\left(x_{2}\right)}=1.2691179 .
\end{aligned}
$$

Hence the conditions of the lemma are satisfied. This means that $\phi\left(x_{1}\right)=F\left(x_{1}\right)<0$ or $\phi\left(x_{2}\right)=F\left(x_{2}\right)<0$ for all choices of $A, B \geq 0$ and consequently the function $f$ defined by (2.2) cannot be in the class $E$, if $T=3.18782$, for any choices of $A \geq 0$ and $B \geq 0$.

REMARK. The choices of $T$ and $A$, in part ( 1 ) of the theorem are not intrinsically significant. Another computer program was set up which, for any given $\mathrm{T}, \mathrm{A}$ and given interval $\left[x_{1}, x_{2}\right]$, could quickly check the values of the function at several points in the interval and calculate relevant quantities in the lemma. The actual T,A,B were then found simply by trial and error by taking some value of $\mathrm{T}$ and eliminating possible choices of A,B by applying the lemma. If for a given $T$ all A,B got eliminated, then a lower $\mathrm{T}$ was chosen. Finally, all calculations were rechecked as before for the chosen $T, A, B$.

The fact that the computer used for this process (Radio Shack TRS 80) was interactive and totally dedicated was very vital in the success of this trial and error method.

ACKNOWLEDGEMENT. The authors are grateful to Professors J.D. Buckholtz and S.Y. Trimble for many helpful discussions, and to the referee for minor corrections and some suggestions.

\section{REFERENCES}

1. LACHANCE, M. Remark of functions with all derivatives univalent, Inter. J. of Math. and Math. Sciences 3 (1980), 193-196.

2. SHAH, S.M. and TRIMBLE, S.Y. Univalent functions with univalent derivatives, Bul1. Amer. Math. Soc. 75 (1969), 153-157.

3. SHAH, S.M. and TRIMBLE, S.Y. Analytic functions with univalent derivatives, Indian J. of Math. 20 (1978), 1-35.

4. TRIMBLE, S.Y. An extension of close-to-convex functions with an application to entire functions with univalent derivatives, to appear. 


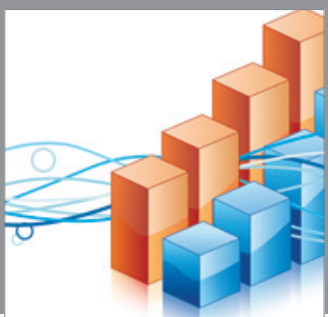

Advances in

Operations Research

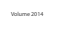

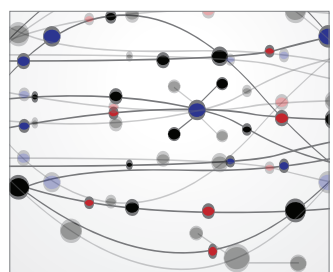

\section{The Scientific} World Journal
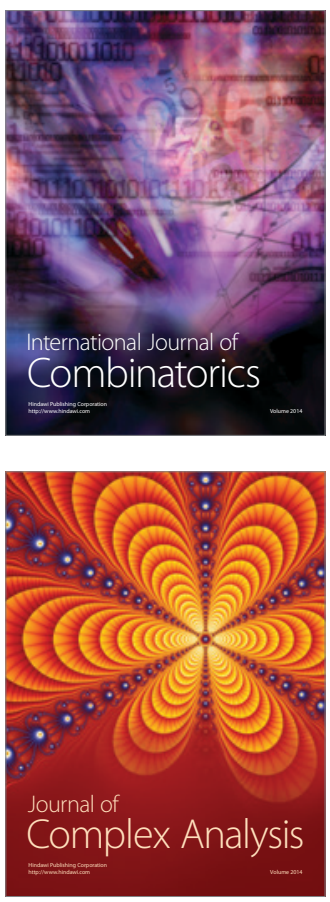

International Journal of

Mathematics and

Mathematical

Sciences
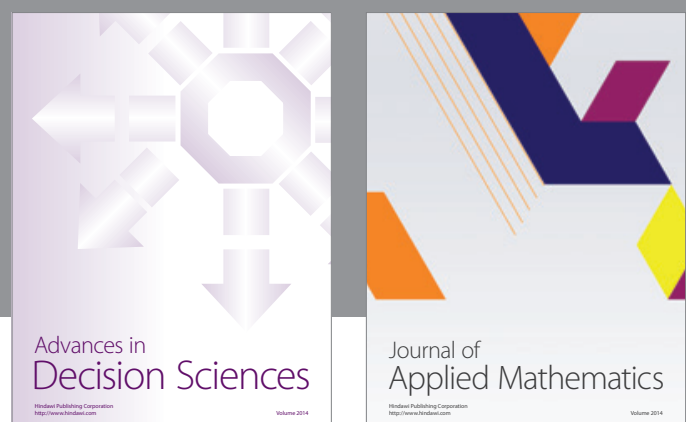

Journal of

Applied Mathematics
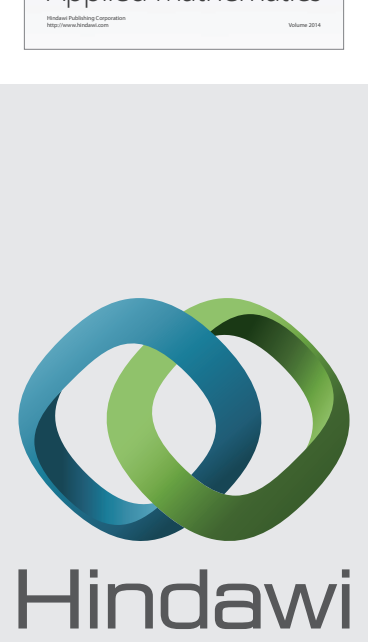

Submit your manuscripts at http://www.hindawi.com
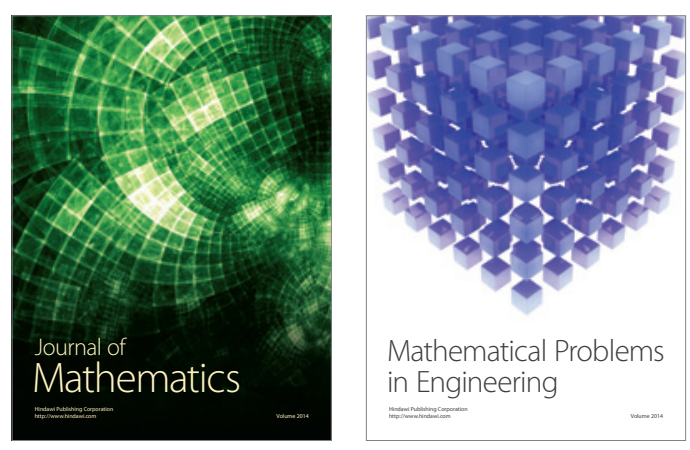

Mathematical Problems in Engineering
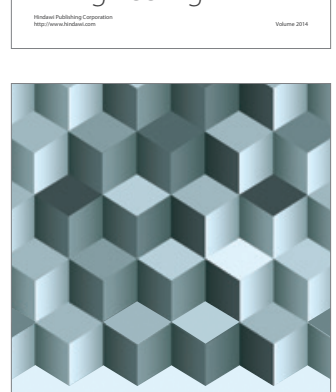

Journal of

Function Spaces
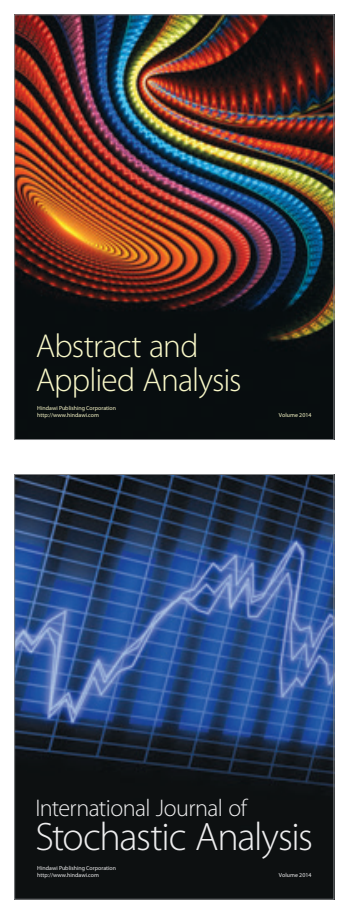

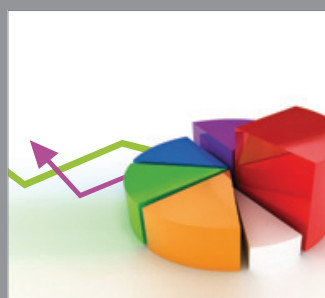

ournal of

Probability and Statistics

Promensencen
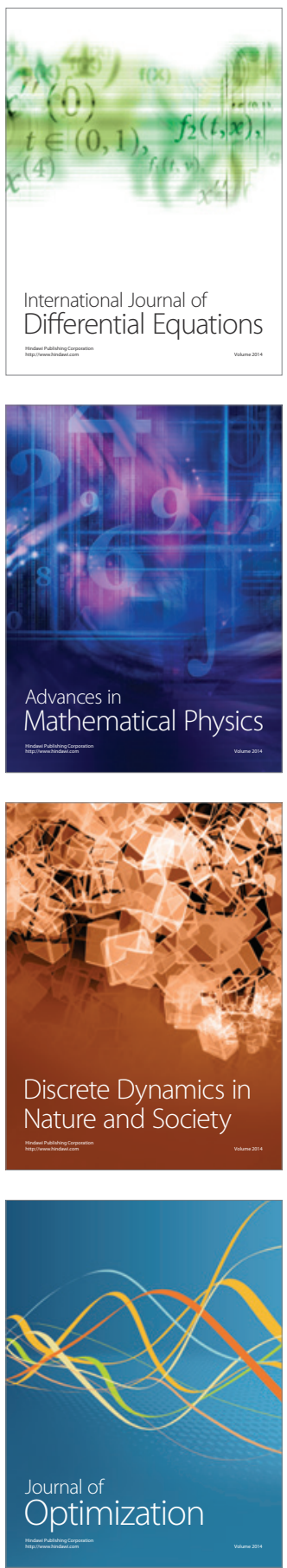